\title{
Drug-related problems identified during pharmacy intervention and consultation: Implementation of an intensive care unit pharmaceutical care model
}

\author{
Xiao-Xiao LI ${ }^{1}$, Si-qian Zheng ${ }^{1}$, Jia-hui $\mathrm{Gu}^{2}$, Tao Huang ${ }^{2}$, Fang Liu ${ }^{1}$, Qing-gang Ge ${ }^{1}$, Bin \\ $\mathrm{Liu}^{1}$, Chao $\mathrm{Li}^{1}$, Min Yi ${ }^{1}$, You-fa Qin ${ }^{3}$, Rongsheng Zhao ${ }^{1}$, and Luwen $\mathrm{Shi}^{2}$ \\ ${ }^{1}$ Peking University Third Hospital \\ ${ }^{2}$ Peking University School of Pharmaceutical Sciences \\ ${ }^{3}$ Affiliated Dongguan Shilong People's Hospital of Southern Medical University
}

June 1, 2020

\begin{abstract}
Aim To identify common drug-related problems (DRPs) during pharmaceutical intervention and consultation in an intensive care unit (ICU); to explore the gap between physicians and pharmacists on their understanding of each other's capabilities and needs. Method A single-center prospective study was conducted in the ICU of a tertiary academic hospital for 21 months. A pharmaceutical care (PC) model was implemented by pharmacy care team, and data was collected during pharmaceutical intervention and consultation. Data analysis was performed on identified DRPs, causes and their relationships. DRPs' frequency during intervention and consultation was compared. Problem-level descriptive analysis and network analysis were conducted using R 3.6.3. Result Implementation of PC model greatly improved the efficacy of pharmacists in both interventions proposed to solve DRPs (from 13.6 to 20.1 cases per month) and number of patients being closely monitored (from 7.7 to 16.9 per month). Pharmacists identified 427 DRPs during pharmaceutical intervention with primarily adverse drug events (ADEs, 34.7\%) and effect of treatment not optimal (25.5\%), and 245 DRPs during consultation (mainly ADEs, 58.4\%). About three-fifths DRPs were caused by antibiotics. Comparing DRPs identified during pharmaceutical intervention and consultation, physicians consulted pharmacists more on questions related to medication safety, while pharmacists also paid attention to treatment effectiveness being consulted less commonly. Conclusion Implementation of PC model is beneficial in guiding pharmacy practice and improving efficacy especially under limited human resources. Physicians and pharmacists shall continue ensuring drug safety and be familiar with the scope of PC and clinical need for a better cooperation.
\end{abstract}

\section{INTRODUCTION}

Since the publication of the report Harvard Medical Practice study [1, 2], drug related problems (DRPs) that might lead to adverse drug events (ADEs) have received extensive attention among the public and healthcare system worldwide. The observed rate of DRP was about 5.6 per 100 patient admissions, with almost half of DRPs being potentially preventable [3]. DRPs also place a substantial health and economic burden on patients and the healthcare system, which cost $\$ 2.8^{\sim} 5.6$ million annually for a 700-bed teaching hospital [1, 4]. Patients admitted to the intensive care unit (ICU) are at higher risk for DRPs [1, 2], primarily cause by their critical diseases status or complications, the use of various high alert medications and the rapid changing pharmacotherapies [5].

Previous studies have shown that critical care pharmacists can play an essential role in promoting the delivery of pharmaceutical care (PC) and improve the overall quality of healthcare by offering individualized recommendations in complicated drug regimens, reducing the incidence rate of DRPs and decreasing preventable ADEs [6-8]. However, the development of PC in the ICU is currently facing three major challenges 
worldwide. Firstly, only a few guidelines provided recommendations on delivery of PC to critically ill patients with fundamental, desirable and optimal pharmacy services and requirements for relative personnel [9]. Secondly, imbalance exists in the development of PC among different specialties and various regions [6]. Last but not least, while the pharmacy profession is widely recognized and pharmacists have become an essential member of the multidisciplinary team (MDT) [10,11], a gap still exists between physicians and pharmacists on their understanding of each other's capabilities and needs. It forms a virtual barrier, and prevents both sides from forming a deep cooperative relationship, even in areas with developed PC system.

Additionally, the ICU PC development situation in China is even more complicated by the relatively late introduction of the concept and limited human resources. Being first advocated in the United States in 1950s [12], the concept of PC and clinical pharmacy was not introduced to China until 1990s [13]. Research in this area is scarce and only a few studies have been published to discuss DRPs. Moreover, while the United States has 14.9 hospital pharmacists available per 100 hospital beds [14], the number was estimated to be 1.4 2.4 in China [15]. Limited human resources made it difficult for pharmacists to provide a comprehensive and daily on-ward-participation of MDT. Instead, some clinical pharmacists could only focus on off-ward services such as therapeutic drug monitoring (TDM), on-call duty for consultations, reevaluation of the prescriptions and pharmaceutical information services $[16,17]$. Thus, a standard pharmacy practice model is needed to promote the development of ICU PC, and a discussion on the classifications and incidence of DRPs is necessary to guide the future efforts in reducing the incidence of DRPs.

We therefore developed a PC model that was tailored to our surgical intensive care unit (SICU) setting, and conducted a prospective study to explore the following questions: (1) Can this PC model guide pharmacists' daily practice properly and help pharmacists identify patients in greater need of PC under limited human resource? Would it potentially improve the efficacy of ICU pharmacists? (2) What are the most common DRPs in the ICU and what are the causes? (3) Is there any difference of DRPs identified during pharmaceutical intervention (offered by pharmacists) and consultation (requested by physicians)? We hope that the answer to this question can help us achieve a better understanding of physicians' and pharmacists' needs and capabilities, and provide a new angle for deepening their bilateral cooperative relationship.

\section{METHOD}

\section{Setting and Study Design}

This is a single-center prospective study conducted in the SICU of a 1891-bed class A tertiary academic hospital, located in Beijing, China, with a duration of 21-month from January, 2018 to September, 2019. This SICU has 19 open beds to admit primarily patients within the hospital for perioperative management, caring for 101 patients per month, with average case-mix index of 4.2 and an overall mortality of $3.8 \%$. One patient on the unit have 5 to 6 medication existing orders per day including 3 to 4 new orders. Three quarters of the patients were from general surgery, urology, gynecology and obstetrics, orthopedic and neurosurgery departments. This study was approved by the Ethical Review Board of Peking University Health Science Center, and informed consent of participant was exempted (IRB00001052-20014).

\section{Pharmaceutical care services provided in SICU}

The content of SICU Pharmaceutical Care Model In this study, PC team used a previously developed PC model to guide pharmacy practice, which was specifically designed for the SICU based on previous experience of 1.5 years on the unit. The PC team consists of one leading pharmacist, a master student in clinical pharmacy major, and/or a pharmacist on clinical pharmacy training. Critical care guidelines and books [18-27] and the leading pharmacist's nutrition background were considered when setting up the care model. One attending doctor participated in the model design, and the deputy director of the SICU department approved it for implementation.

The PC model defined the high-risk patient population and drug class that need priority monitoring, with an additional list of pharmacy services that should be provided to ensure medication safety. Key points were provided to guide specific interventions for each high-risk patient population, drug class and pharmacy service 
mentioned, see Supplementary materials for the complete PC model. High-risk patient population includes patients with chronic renal disease, reduced liver function or other baseline chronic disease, and patients with unstable hemodynamics, severe infections, obesity or pregnancy, etc. High-risk medications include enteral and parenteral nutrition therapy, antibiotics, anti-coagulants, drugs come with unique dosage forms, etc. Pharmaceutical services listed include prescription verification, TDM, identification of adverse drug reactions (ADRs) and drug-drug interactions, and providing drug information and clinical consultations.

SICU Pharmaceutical Care Model Implementation During the study period, the care team used the PC model as a guide to identify patients and medications which need prioritizing pharmaceutical monitoring. For example, if a patient has reduced renal function, the severity and cause of renal dysfunction should be assessed by the pharmacist. Then, the medication regimen should be reviewed daily and dose adjustment recommendations should be made if needed; close monitoring of the patient's fluid balance and serum creatinine clearance should also be performed. For patients on antibacterial medications, infection should first be confirmed by verifying patient clinical symptoms, lab values, together with results of imaging and etiological examinations. And drug regimen should be evaluated for safety and effectiveness.

On a daily basis, the PC team attends shift meeting and rounding in the morning, and spend 2.5-3.5 hours a day on average at the SICU unit. The pharmacists will check new prescription orders of the patients mentioned in the shift meeting and the team they rounded with, and make face-to-face communications if any change needs to be made. For the rest of the time (4.5-5.5 hours a day on average), the care team will be at the pharmacy department, and communications can be made through phone calls or a social software called WeChat . The care team also attends the weekly case discussion scheduled on every Wednesday. It usually covers one to two complicated patient cases, including dead cases if applicable.

\section{Data collection and classification}

Data was collected by the leading pharmacist after providing pharmaceutical interventions and completing consultations. The information collected mainly includes case number, patient gender, description of the DRP, pharmacists' recommendations, the consultation questions and answers, etc.

During DRP classification, the Pharmaceutical Care Network Europe (PCNE) Classification system was initially chosen for it considered as a validated system for DRP classification in hospital settings [28, 29]. However, during the pilot study, we found that certain DRPs and their related causes were not included in the system. We therefore added a few columns and used a modified PCNE V9.0 Classification system for DRP analysis after reaching a consensus among all three members participated in this process, see Supplementary materials . For example, P3.3 was added for need additional drug therapy monitoring, and C1.8 was added for necessary genetic testing before drug initiation (for drugs such as carbamazepine). The classification system for pharmaceutical consultations was also created based on the original PCNE V9.0 Classification system using a similar strategy with pharmaceutical intervention classification.

During DRP classification, as shown in Figure 1, a pilot test was performed and DRPs collected were classified by two researchers separately using the modified PCNE V9.0 classification system. A third researcher would make the final decision if classification results unmatched. The consultation was classified using the same method (see Supplementary materials ). In addition, the classification of medication errors (ME) was conducted using the National Coordinating Council for Medication Error Reporting and Prevention (NCC-MERP) classification criteria for pharmaceutical intervention [30].

\section{Statistical analysis}

Problem-level descriptive analysis was used to characterize the DRPs and relevant causes and interventions. Monthly intervention rate, and monthly average number of interventions per patient were calculated. Network analysis were performed to find the potential causes led to specific drug related problems during pharmaceutical intervention and consultation separately. Additionally, DRPs identified during pharmaceutical intervention and consultation were compared in the purpose of discovering similarities and differences of the focus on medication use from the perspective of physicians and pharmacists. Categorical variables were 
described using frequency counts and percentages, and continuous variables were described using medians with interquartile range (IQR). All the calculation and analysis were conducted using R 3.6.3.

\section{RESULT}

\section{Basic information and efficacy evaluation}

During the 21-month study, the pharmacy care team performed close monitoring to a total number of 354 patients identified by using SICU PC model. The average age of the patient population was 57 years old (IQR 41,76$)$ and $56.8 \%$ of them were male $(n=201)$. According to the model, these patients need priority monitoring as they have baseline diseases of renal dysfunction (including patients on renal replacement therapy; $19.2 \%, n=68)$, reduced heart function or blood pressure instability $(11.3 \%, n=40)$, coagulopathy $(10.5 \%, n=37)$, or liver dysfunction $(9.3 \%, n=33)$; or they are on key medications such as antibiotics $(61.6 \%, n=218)$ and nutrition support therapy $(20.6 \%, n=73)$, or medications required TDM $(20.3 \%, n$ $=72)$, etc.

In this period, 427 DRPs were identified and 486 pharmaceutical interventions were proposed by pharmacists to solve the problems during priority monitoring; 245 DRPs were identified and 273 interventions related to drug therapy changes were proposed during consultation. For additional pharmacy services, the team made 93 individualized drug regimen recommendations for TDM patients or based on genetic test results, reported 21 cases of ADR, and provided 13 teaching sessions to the physicians and/or nurses in the SICU.

We compared the pharmacy-related services completed during 2017 (before PC model implementation) and the study period to assess if its implementation could improve the efficacy of PC team in providing patient care services. Under the guidance of the SICU PC model, the number of patients on closely monitoring by the PC team increased from 7.7 to 16.9 per month. The monthly pharmaceutical interventions made increased from 13.6 to 20.1 cases, while the consultations provided slightly decreased from 13.3 to 12.6 cases (see Supplementary materials ).

\section{Pharmaceutical Intervention}

During the close monitoring of 354 SICU patients, 423 pharmaceutical intervention records were collected and pharmaceutical interventions were suggested for 213 patients (60.2\%). A total number of 427 DRPs were identified from 412 records (11 records related to non-medical problems were removed, see Figure 1 ), and pharmacists proposed 486 interventions to solve the DRPs with an acceptance rate of $97.3 \%$. On average, interventions were made to 12.1 cases per 100 patient cases admitted to the unit; pharmaceutical interventions were proposed for $34.9 \%$ patients who are on priority monitoring, and 0.9 recommendations were made for each patient on the unit per month.

ME classification ME classification using NCC-MERP criteria was performed for the 427 DRPs identified during pharmaceutical intervention. Most of the DRPs were classified as category C $(82.2 \%, \mathrm{n}=351)$, followed by category $\mathrm{D}(7.5 \%, \mathrm{n}=32)$ and $\mathrm{E}(5.6 \%, \mathrm{n}=24)$, with the latter two causing potential harms or harms to patients. MEs in category A and B only counted for $4.7 \%$ of the total ( $\mathrm{n}=5,15$ respectively).

DRPs and pharmaceutical interventions during close patient monitoring Data analysis results of DRPs and Causes related to all medications and antibiotics were shown in Table 1 . Among 427 DRPs identified from all medications, the primary problems were treatment safety and effectiveness ( $69 \%$ in total), including "P2.1 Adverse drug event (possibly) occurring", "P1.2 Effect of drug treatment not optimal", and "P3.2 Unnecessary drug-treatment". Medication classification results indicated that the top 3 medicines leading to DRPs were antibiotics (59.7\%), parenteral nutrition (5.8\%) and proton pump inhibitors (PPI, 2.7\%), see Supplementary materials for details.

During the 21-month study period, a total number of 486 interventions were proposed by pharmacists to solve the DRPs. About four fifths $(81.7 \%)$ of the interventions were made at drug level, mainly including "I3.5 Drug paused or stopped" (27.4\%), "I3.2 Dosage changed" (26.5\%) and "I3.1 Drug changed" (15.8\%), see Supplementary materials . Less than one fifth (17.9\%) interventions (such as ordering labs or genetic 
tests) were made at prescriber level, of which $98 \%$ were proposed to the prescriber. Only $0.4 \%$ interventions were proposed at patient level as most of the SICU patients were sedated and not able to communicate.

Causes of identified DRPs The analysis of 479 DRP causes of all medications showed "C1 Drug selection" caused the highest proportion of DRPs (41.3\%), followed by "C3 Dose selection" and "C4 Treatment duration". The major sub-category of DRP causes were "C4.2 Duration of treatment too long", "C3.2 Drug dose too high", and "C9.1 No or inappropriate outcome monitoring (incl. TDM)" (see Supplementary materials ). The relationship between the DRPs and Causes was analyzed and shown in Figure 2. The relationship between the DRPs and Causes in antibiotic use was shown in Supplementary materials .

\section{Pharmaceutical Consultation}

During the 21-month study period, 265 pharmaceutical consultation records were collected, and recommendations related to drug therapy changes were suggested for 131 patients, including 9 children and 5 prenatal women. The mean age of the patient population was 61 (IQR 42, 74) years old, and $58.1 \%$ of patients were males $(n=105)$. Notably, $29 \%$ of the patients have renal dysfunction. Only 243 records were included for DRP classification (22 records on non-clinical issues or from non-SICU departments removed, see Supplementary materials ), from which 245 DRPs were identified. A total number of 273 interventions related to drug therapy changes were proposed by pharmacists (consultations provided drug information only were not counted) with an acceptance rate of $99.3 \%$. On average, pharmacists completed 12.6 consultations and proposed 13 interventions related to drug therapy changes per month.

DRPs and Causes identified during pharmaceutical consultation Data analysis results of DRPs and Causes related to all medications and antibiotics were shown in Table 2. Among 245 DRPs identified during pharmaceutical consultation, the proportion of "P2 Treatment safety" (58.4\%) was significantly higher than others. The major sub-category of DRPs were "P2.1 Adverse drug event (possibly) occurring", "P1.3 Untreated symptoms or indication", and "P1.2 Effect of drug treatment not optimal". The medication classification results of all DRPs indicated the top 3 medicines being consulted were antibiotics $(62.2 \%$, $\mathrm{n}=153)$, antifungal drugs $(8.53 \%, \mathrm{n}=21)$, and antiepileptic drugs $(3.66 \%, \mathrm{n}=9)$, see Supplementary materials for details.

The analysis result of 246 DRP Causes showed "C3 Dose selection" caused the highest proportion of DRPs (59.8\%), followed by "C1 Drug selection" and "C7 Drug-related side effects/Drug-induced diseases". The relationship between DRPs and Causes was analyzed and shown in Figure 2 . For the relationship between DRPs and Causes in antibiotic use, see Supplementary materials .

\section{Comparison of (possible) DRPs identified during pharmaceutical interventions and consulta- tions}

In this session, we compared 4 sub-categories of DRPs as they have the same definition in pharmaceutical intervention and consultation classification. As shown in Figure 3, two bubbles representing untreated symptoms or indications and adverse drug events respectively are above the oblique line, which indicated physicians tend to ask pharmacists for help when they have questions in these two areas. The other two bubbles below the line representing drug treatment showing no effect or effect not optimized, which indicated pharmacists tend to solve more DRPs in these two areas than they are being asked by physicians. "P3.1 Problem with cost-effectiveness of the treatment" and "P3.2 Unnecessary drug-treatment" were incomparable because of their appearance in pharmaceutical intervention only.

\section{DISCUSSION}

In this prospective study, we showed a dedicated pharmacy care team in the SICU could work more efficiently in patient monitoring and identifying DRPs and preventable MEs though implementating a PC model. The number of pharmacutical interventions proposed on medication orders increased from 13.6 cases monthly to 20.1 cases monthly, and the patients on priority monitoring by the PC team doubled.

The primary results of our study are in line with previous reports. (1) The incidence rates of interventions 
is 12.1 cases per 100 patient admissions, being similar to the rate of preventable ordering adverse drug events as 14.7 per 100 patients in previous report [10]. (2) ADE occurring (31.1\%) was the most frequently detected DRPs and more than four fifths of DRPs were due to drug selection, dose selection and treatment duration, being in line with an evaluation of pharmacists' interventions in a Swiss study [8]. (3) Drugs that most frequently caused DRPs during intervention were antibiotics, showing a similar composition but with a higher percentage than that of previous studies [8,31,32]. (4) More than $97 \%$ of advice given by the pharmacist were accepted or taken into consideration, being much higher than previously reported in a mixed Norwegian ICU (87\%) [31], but the same as it in a Swiss university hospital (97.8\%) [8]. Though the priority monitoring was only performed for one-fifths of SICU patients due to limited human resources, the primary results of this study were consistent with other studies covering the total patients. It suggests that the PC model implemented in this study is efficient in identifying high-risk patient population that pharmacists should focus on; and the establishment of such practice models can be beneficial to institutions under limited human resources in identifying high-risk patients and the majority of DRPs.

There are two important findings in this study, and the first one is related to antibiotic use. Similar to previous reports, we found antibiotics was the top medicine related DRPs during both pharmaceutical intervention $(59.7 \%)$ and consultation $(62.2 \%)$. This can be primarily explained by the high prevalence of infectious diseases in ICU setting [8,31,32]. Moreover, our study showed a much higher proportion of DRPs caused by antibiotics (comparing with $48.9 \%$ in a Swiss ICU [8] and $22 \%$ in a mixed Norwegian ICU [31] ), which can be explained partly by the current antibiotic management strategies in China. A series of measures were adopted by Chinese government to improve antibiotic use over the past decades [33, 34], including the establishment of national guidelines [20,27], the surveillance networks for antibiotic use and antimicrobial resistance [35], and a 3-year national level regulatory campaign launched in 2011 [36]. Following these regulations, an antibiotic administrative group was set up in the studied hospital for antibiotic use monitoring, and they announce the drug utilization and re-evaluation results of antibiotics prescription monthly. The high pressures from governmental policies, together with hospital internal management, made both physicians and pharmacists be more conscious of antibiotic use.

Additionally, among the total 286 DRPs related to antibiotics during intervention, the most frequent causes were dose selection, inappropriate outcome monitoring and inappropriate drug according to guidelines, indicating the weakness of physicians' knowledge in such areas. In practice, clinical pharmacists could fill the gap by working as an integral member in the MDT and offering optimal antimicrobial therapies according to infection sites, pathogens, pharmacokinetics/ pharmacodynamics (PK/PD) parameters of antibiotics, and patients' renal or liver function [37, 38]. In addition to consulting pharmacists on antibiotic selection and dosing, it may also be important to set up more targeted courses to equip ICU physicians with essential knowledge on antibiotic use [39, 40].

The second important findings was the difference of identified DRPs between pharmaceutical interventions and consultations. Though comparison, we found that SICU physicians tend to seek recommendations actively from clinical pharmacists in face of identifying ADRs, choosing a cost-effectiveness treatment, and requiring a dosage regimen when a new therapy initiates. However, under circumstances as selecting a drug for untreated symptoms or indications, optimizing therapy or discontinuing unnecessary treatment, SICU physicians tend to accept recommendations passively from clinical pharmacists.

There were three possible reasons for this. Firstly, with a better understanding of diseases and the result of experimental teaching, SICU physicians more rely on their accumulated clinical experience instead of updated guidelines that clinical pharmacists attach importance to when evaluating treatment effectiveness. Secondly, owing to the legal considerations of off label-use [41], and the uncertainty on the applicability and safety of dosage recommendations of international guidelines on local populations [42, 43], SICU physicians are prone to a therapeutic option with approved indications and dosage regimens, even if there is a potential better option. Thirdly, in the selected SICU where most patients were in perioperative period, it is common for the operators to participate in making clinical decisions as consultants or co-attendings. ICU physicians may feel pressure when they hold different opinions on treatment plans, and unnecessary medication orders 
can be carried out in this way. These situations bring great opportunities for pharmacists to get involved, communicate with both sides and ensure medication safety [7-9, 44, 45].

At present, pharmacists have been recognized by physicians as an essential member of the MDT, but fully understanding of each other's skills and needs remains incomplete is still needed. SICU physicians should learn more about pharmacist capabilities in drug selection and regimen design, and pharmacists should continue improving their knowledge on therapeutics and medication safety to support physicians better. Finally, ADE occurring were the most frequent DRPs both in pharmaceutical intervention and consultation. It suggests that both physicians and pharmacists should continue strengthening a close cooperation in drug safety.

The study has three strengths. To start with, it is the first study to evaluate an ICU PC model in China. We carried out a prospective study and enrolled 354 consecutive SICU patients under this model, which was developed by an experienced pharmacist team. According to the results, it may generally applied to other ICU departments, especially those under limited human resources. Secondly, the DRP classification was completed by three researchers to guarantee the accuracy. Two of them took responsibility for the categorization of DRP types and subtypes, and a pilot test was performed to ensure they have the same understanding of the classification system. Thirdly, our study creatively compared the DRPs identified during pharmaceutical interventions and consultations, and showed the gap between pharmacy services currently being provided and the needs of physicians. The result will not only help physicians better understand the scope of pharmacy service beyond drug safety, but also guide pharmacists during their daily practice by reassuring the clinical needs of physicians.

The study also has two limitations. Firstly, data collection was mostly performed by the leading pharmacist, which may lead to information bias and the possibility of underestimating DRPs incidence. Nevertheless, three researchers participated in the DRP classification to minimize the bias of the study results. Secondly, the original DRP system cannot cover every PC point; we therefore developed a modified DRP system with slight changes to capture the key DRP types and causes.

\section{CONCLUSIONS}

This study revealed the most common DRPs in a Chinese SICU setting during pharmaceutical interventions and consultations. Our results indicate that the establishment and implementation of an ICU PC mode is beneficial for guiding pharmacy practice and improving efficacy especially when human resource is limited. Additionally, physicians and pharmacists should continue their efforts in ensuring drug safety and get a better understanding of the scope of PC practice and clinical need in order to achieve a deeper cooperation in MDTs and improve the quality of ICU patient care together in the long run.

\section{AUTHORS' CONTRIBUTIONS}

All authors took part in the final version for submission and accept overall accountability for accuracy and integrity of the manuscript.

LXX: Conceptualization, methodology, investigation, writing - original draft

ZSQ: Conceptualization, methodology, data curation, writing - original draft

GJH: Data curation, ethics application

HT: Formal analysis, visualization

LF: Supervision, Writing - review \& editing

GQG : Supervision

LB: Investigation

LC: Investigation 
YM: Supervision

QYF: Data curation

ZRS: Supervision, validation, funding acquisition

SLW: Supervision, validation, funding acquisition

\section{ACKNOWLEDGEMENTS}

We thank Associate Prof. Xiao-dong Guan (Department of Pharmacy Administration and Clinical Pharmacy, School of Pharmaceutical Sciences, Peking University) and Dr. Hua Zhang (Research Center of Clinical Epidemiology, Peking University Third Hospital ) for the help of study design. We are also grateful for all the physicians and nurses inCritical Care Medicine Department of Peking University Third Hospital for the research assistance, including Prof. Peng-lin Ma, Prof. Gai-qi Yao, Prof. Xi Zhu, Associate Prof. Qiang Li, Senior Doc. Zong-yu Wang, Senior Doc. Tie-hua Wang, Senior Doc. Zhi-ling Zhao, Doc. Jing-jing Xi, Chief resident Yong-shi Han and the head nurse Xian-jie Cui. We thank graduate students in Clinical Pharmacy, School of Pharmaceutical Sciences,Peking University for supporting this work, including $\mathrm{Na} \mathrm{He}$, Chu-hui Wang, Shan Su, Jie-yu Zhao and Yi Chen. Additional thanks to Yang Zhao (Medicine School of Lanzhou University) and Peng Yao (International Research Center for Medicinal Administration, Peking University ), for their recommendations on article writing and editing.

\section{CONFLICT OF INTEREST STATEMENT}

The authors declare that they have no competing interests.

\section{FUNDING INFORMATION}

This work was supported by the National Science and Technology Major Project of China (grant number 2018ZX09721003-001-002, 2018 and 2017ZX09304012, 2017).

\section{AVAILABILITY OF DATA AND MATERIALS}

The statistical report analysed during this study are included in this published article and its supplementary information files. The datasets used during the current study are available from the corresponding author on reasonable request.

\section{ETHICS APPROVAL AND CONSENT TO PARTICIPATE}

This study was approved by the Ethical Review Board of Peking University Health Science Center, and informed consent of participant was exempted (IRB00001052-20014).

\section{REFERENCE}

1. Brennan T A, Leape L L, Laird N M, Hebert L, Localio A R, Lawthers A G, Newhouse J P, Weiler P $\mathrm{C}$, Hiatt $\mathrm{H} \mathrm{H}$. Incidence of adverse events and negligence in hospitalized patients. Results of the Harvard Medical Practice Study I. N Engl J Med 1991; 324(6): 370-376.

2. Leape L L, Brennan T A, Laird N, Lawthers A G, Localio A R, Barnes B A, Hebert L, Newhouse J P, Weiler P C, Hiatt H. The nature of adverse events in hospitalized patients. Results of the Harvard Medical Practice Study II. N Engl J Med 1991; 324(6): 377-384.

3. Leendertse A J, Egberts A C, Stoker L J, van den Bemt P M, Group H S. Frequency of and risk factors for preventable medication-related hospital admissions in the Netherlands. Arch Intern Med 2008; 168(17): 1890-1896.

4. Bates D W, Spell N, Cullen D J, Burdick E, Laird N, Petersen L A, Small S D, Sweitzer B J, Leape L L. The costs of adverse drug events in hospitalized patients. Adverse Drug Events Prevention Study Group. JAMA 1997; 277(4): 307-311. 
5. Kari H, Kortejarvi H, Airaksinen M, Laaksonen R. Patient involvement is essential in identifying drugrelated problems. Br J Clin Pharmacol 2018; 84(9): 2048-2058.

6. Lee H, Ryu K, Sohn Y, Kim J, Suh G Y, Kim E. Impact on Patient Outcomes of Pharmacist Participation in Multidisciplinary Critical Care Teams: A Systematic Review and Meta-Analysis. Crit Care Med 2019; 47(9): 1243-1250.

7. Tasaka Y, Tanaka A, Yasunaga D, Asakawa T, Araki H, Tanaka M. Potential drug-related problems detected by routine pharmaceutical interventions: safety and economic contributions made by hospital pharmacists in Japan. J Pharm Health Care Sci 2018; 4: 33.

8. Reinau D, Furrer C, Stampfli D, Bornand D, Meier C R. Evaluation of drug-related problems and subsequent clinical pharmacists' interventions at a Swiss university hospital. J Clin Pharm Ther 2019; 44(6): 924-931.

9. Rudis M I, Brandl K M. Position paper on critical care pharmacy services. Society of Critical Care Medicine and American College of Clinical Pharmacy Task Force on Critical Care Pharmacy Services. Crit Care Med 2000; 28(11): 3746-3750.

10. Leape L L, Cullen D J, Clapp M D, Burdick E, Demonaco H J, Erickson J I, Bates D W. Pharmacist participation on physician rounds and adverse drug events in the intensive care unit. JAMA 1999; 282(3): 267-270.

11. Zhou X, Qiu F, Wan D, Sun S, Yao G, Liu Y, Li J. Nutrition support for critically ill patients in China: role of the pharmacist. Asia Pac J Clin Nutr 2019; 28(2): 246-251.

12. Anderson R D. Mirror to ASHP: 1942-1992. Am J Hosp Pharm 1992; 49(8): 1925-35.

13. Hu M, Yee G, Zhou N, Yang N, Jiang X, Klepser D. Development and current status of clinical pharmacy education in China. Am J Pharm Educ 2014; 78(8): 157.

14. Schneider P J, Pedersen C A, Ganio M C, Scheckelhoff D J. ASHP national survey of pharmacy practice in hospital settings: Workforce-2018. Am J Health Syst Pharm 2019; 76(15): 1127-1141.

15. Li X X, Zhang C, Liu F, Zhai S D, Yang Y H. Application of PDCA cycle method in the establishment and implementation of quality control system in clinical pharmacy. Chin J Hosp Pharm 2019; 39(22): 2347-2350.

16. Wei Y H, Shi L W, Shao H, Nie X Y, Li X X, Yang W, Wang N, Zheng K, Bao N J, Wu Y P. Current status and analysis of Chinese clinical pharmacists. Chin J New Drug 2011; 20(9): 844-848.

17. Penm J, Moles R, Wang H, Li Y, Chaar B. Factors affecting the implementation of clinical pharmacy services in China. Qual Health Res 2014; 24(3): 345-356.

18. Chou R, Gordon D B, de Leon-Casasola O A, Rosenberg J M, Bickler S, Brennan T, Carter T, Cassidy C L, Chittenden E H, Degenhardt E, Griffith S, Manworren R, McCarberg B, Montgomery R, Murphy J, Perkal M F, Suresh S, Sluka K, Strassels S, Thirlby R, Viscusi E, Walco G A, Warner L, Weisman S J, Wu C L. Management of Postoperative Pain: A Clinical Practice Guideline From the American Pain Society, the American Society of Regional Anesthesia and Pain Medicine, and the American Society of Anesthesiologists' Committee on Regional Anesthesia, Executive Committee, and Administrative Council. J Pain 2016; 17(2): 131-157.

19. Frontera J A, Lewin J J, 3rd, Rabinstein A A, Aisiku I P, Alexandrov A W, Cook A M, Del Zoppo G J, Kumar M, Peerschke E I, Stiefel M F, Teitelbaum J S, Wartenberg K E, Zerfoss C L. Guideline for Reversal of Antithrombotics in Intracranial Hemorrhage: Executive Summary. A Statement for Healthcare Professionals From the Neurocritical Care Society and the Society of Critical Care Medicine. Crit Care Med 2016; 44(12): 2251-2257. 
20. Ministry of Health. Notification on issuing National Guiding Principles for Antimicrobial Clinical Use. [OB/EL] 2004 2004-08-19 17 May 2020]; Available from: http://www.nhc.gov.cn/wjw/gfxwj/201304/2c850f3dc54244ca846d8a17baf3613d.shtml.

21. Kellum J A, Lameire N, Group K A G W. Diagnosis, evaluation, and management of acute kidney injury: a KDIGO summary (Part 1). Crit Care 2013; 17(1): 204.

22. McClave S A, Taylor B E, Martindale R G, Warren M M, Johnson D R, Braunschweig C, McCarthy M S, Davanos E, Rice T W, Cresci G A, Gervasio J M, Sacks G S, Roberts P R, Compher C, Society of Critical Care M, American Society for P, Enteral N. Guidelines for the Provision and Assessment of Nutrition Support Therapy in the Adult Critically Ill Patient: Society of Critical Care Medicine (SCCM) and American Society for Parenteral and Enteral Nutrition (A.S.P.E.N.). JPEN J Parenter Enteral Nutr 2016; 40(2): 159-211.

23. Rhodes A, Evans L E, Alhazzani W, Levy M M, Antonelli M, Ferrer R, Kumar A, Sevransky J E, Sprung C L, Nunnally M E, Rochwerg B, Rubenfeld G D, Angus D C, Annane D, Beale R J, Bellinghan G J, Bernard G R, Chiche J D, Coopersmith C, De Backer D P, French C J, Fujishima S, Gerlach H, Hidalgo J L, Hollenberg S M, Jones A E, Karnad D R, Kleinpell R M, Koh Y, Lisboa T C, Machado F R, Marini J J, Marshall J C, Mazuski J E, McIntyre L A, McLean A S, Mehta S, Moreno R P, Myburgh J, Navalesi P, Nishida O, Osborn T M, Perner A, Plunkett C M, Ranieri M, Schorr C A, Seckel M A, Seymour C W, Shieh L, Shukri K A, Simpson S Q, Singer M, Thompson B T, Townsend S R, Van der Poll T, Vincent J L, Wiersinga W J, Zimmerman J L, Dellinger R P. Surviving Sepsis Campaign: International Guidelines for Management of Sepsis and Septic Shock: 2016. Crit Care Med 2017; 45(3): 486-552.

24. Ye Z K, Chen Y L, Chen K, Zhang X L, Du G H, He B, Li D K, Liu Y N, Yang K H, Zhang Y Y, Zhai S D, Guideline Steering Group t G D G, the Guideline Secretary G. Therapeutic drug monitoring of vancomycin: a guideline of the Division of Therapeutic Drug Monitoring, Chinese Pharmacological Society. J Antimicrob Chemother 2016; 71(11): 3020-3025.

25. Borthwick M, Barton G, Bourne R S, McKenzie C. Critical care pharmacy workforce: UK deployment and characteristics in 2015. Int J Pharm Pract 2018; 26(4): 325-333.

26. Taskforce D A S, Baron R, Binder A, Biniek R, Braune S, Buerkle H, Dall P, Demirakca S, Eckardt R, Eggers V, Eichler I, Fietze I, Freys S, Frund A, Garten L, Gohrbandt B, Harth I, Hartl W, Heppner H J, Horter J, Huth R, Janssens U, Jungk C, Kaeuper K M, Kessler P, Kleinschmidt S, Kochanek M, Kumpf M, Meiser A, Mueller A, Orth M, Putensen C, Roth B, Schaefer M, Schaefers R, Schellongowski P, Schindler M, Schmitt R, Scholz J, Schroeder S, Schwarzmann G, Spies C, Stingele R, Tonner P, Trieschmann U, Tryba M, Wappler F, Waydhas C, Weiss B, Weisshaar G. Evidence and consensus based guideline for the management of delirium, analgesia, and sedation in intensive care medicine. Revision 2015 (DAS-Guideline 2015) - short version. Ger Med Sci 2015; 13: Doc19.

27. Ministry of Health. Notification on issuing National Guiding Principles (2015) for Antimicrobial Clinical Use. [OB/EL] 2015 2015-07-24 17 May 2020]; Available from: http://www.nhc.gov.cn/yzygj/s3593/201508/c18e1014de6c45ed9f6f9d592b43db42.shtml.

28. Qu C, Meng L, Wang N, Chen Y, Yang X, Wang J, Sun S, Qiu F. Identify and categorize drug-related problems in hospitalized surgical patients in China. Int J Clin Pharm 2019; 41(1): 13-17.

29. Griese-Mammen N, Hersberger K E, Messerli M, Leikola S, Horvat N, van Mil J W F, Kos M. PCNE definition of medication review: reaching agreement. Int J Clin Pharm 2018; 40(5): 1199-1208.

30. Brixey J, Johnson T R, Zhang J. Evaluating a medical error taxonomy. Proc AMIA Symp 2002: 71-75.

31. Johansen E T, Haustreis S M, Mowinckel A S, Ytrebo L M. Effects of implementing a clinical pharmacist service in a mixed Norwegian ICU. Eur J Hosp Pharm 2016; 23(4): 197-202.

32. Klopotowska J E, Kuiper R, van Kan H J, de Pont A C, Dijkgraaf M G, Lie A H L, Vroom M B, 
Smorenburg S M. On-ward participation of a hospital pharmacist in a Dutch intensive care unit reduces prescribing errors and related patient harm: an intervention study. Crit Care 2010; 14(5): R174.

33. Van Boeckel T P, Gandra S, Ashok A, Caudron Q, Grenfell B T, Levin S A, Laxminarayan R. Global antibiotic consumption 2000 to 2010: an analysis of national pharmaceutical sales data. Lancet Infect Dis 2014; 14(8): 742-750.

34. State Council of China. Opinions of the CPC Central Committee and the State Council on Deepening the Health Care System Reform. [OB/EL] 2009 2009-04-09 17 May 2020]; Available from: http://www.china.org.cn/government/scio-press-conferences/2009-04/09/content_17575378.htm.

35. Ministry of Health. Notification on Establishing China Antimicrobial Resistance Surveillance System and Center for Antibacterial Surveillance. [OB/EL] 2005 2005-11-29 17 May 2020]; Available from: http://www.nhc.gov.cn/zwgk/jdjd/201304/b0da0ebfc7b3428f98d435a29cfa4250.shtml.

36. Ma X, Xie J, Yang Y, Guo F, Gao Z, Shao H, Huang Y, Yang C, Qiu H. Antimicrobial stewardship of Chinese ministry of health reduces multidrug-resistant organism isolates in critically ill patients: a pre-post study from a single center. BMC Infect Dis 2016; 16(1): 704.

37. Wang H, Wang H, Yu X, Zhou H, Li B, Chen G, Ye Z, Wang Y, Cui X, Zheng Y, Zhao R, Yang H, Wang Z, Wang P, Yang C, Liu L. Impact of antimicrobial stewardship managed by clinical pharmacists on antibiotic use and drug resistance in a Chinese hospital, 2010-2016: a retrospective observational study. BMJ Open 2019; 9(8): e026072.

38. Zhou L, Ma J, Gao J, Chen S, Bao J. Optimizing Prophylactic Antibiotic Practice for Cardiothoracic Surgery by Pharmacists' Effects. Medicine (Baltimore) 2016; 95(9): e2753.

39. Guan X, Tian Y, Song J, Zhu D, Shi L. Effect of physicians' knowledge on antibiotics rational use in China's county hospitals. Soc Sci Med 2019; 224: 149-155.

40. Wushouer H, Wang Z, Tian Y, Zhou Y, Zhu D, Vuillermin D, Shi L, Guan X. The impact of physicians' knowledge on outpatient antibiotic use: Evidence from China's county hospitals. Medicine (Baltimore) 2020; 99(3): e18852.

41. Lenk C, Duttge G. Ethical and legal framework and regulation for off-label use: European perspective. Ther Clin Risk Manag 2014; 10: 537-546.

42. Kim N H, So M S, Kang J G, Cho D S, Byrne C D, Lee S J, Sung K C. Application of new guidelines for the primary prevention of atherosclerotic cardiovascular disease in a Korean population. J Atheroscler Thromb 2015; 22(3): 293-303.

43. Koga M, Shiokawa Y, Nakagawara J, Furui E, Kimura K, Yamagami H, Okada Y, Hasegawa Y, Kario K, Okuda S, Endo K, Miyagi T, Osaki M, Minematsu K, Toyoda K. Low-dose intravenous recombinant tissuetype plasminogen activator therapy for patients with stroke outside European indications: Stroke Acute Management with Urgent Risk-factor Assessment and Improvement (SAMURAI) rtPA Registry. Stroke 2012; 43(1): 253-255.

44. Kane S L, Weber R J, Dasta J F. The impact of critical care pharmacists on enhancing patient outcomes. Intensive Care Med 2003; 29(5): 691-698.

45. Penm J, Li Y, Zhai S, Hu Y, Chaar B, Moles R. The impact of clinical pharmacy services in China on the quality use of medicines: a systematic review in context of China's current healthcare reform. Health Policy Plan 2014; 29(7): 849-872.

\section{TABLES}

Table 1 Number of Drug related Problems and Causes of all Medicines and Antibiotics during Pharmaceutical Intervention 


\begin{tabular}{lllll}
\hline Description & All medicines & All medicines & Antibiotic & Antibiotic \\
\hline Problem & N & Proportion, \% & N & Proportion, \% \\
\hline
\end{tabular}

\begin{tabular}{|c|c|c|}
\hline P1 Treatment effectiveness & 146 & 34.2 \\
\hline P1.1 No effect of drug treatment & 14 & 3.3 \\
\hline P1.2 Effect of drug treatment not optimal & 109 & 25.5 \\
\hline P1.3 Untreated symptoms or indication & 23 & 5.4 \\
\hline P2 Treatment safety & 133 & 31.1 \\
\hline P2.1 Adverse drug event (possibly) occurring & 133 & 31.1 \\
\hline P3 Other & 148 & 34.7 \\
\hline P3.1 Problem with cost-effectiveness of the treatment & 3 & 0.7 \\
\hline P3.2 Unnecessary drug-treatment & 89 & 20.8 \\
\hline P3.3 Needs additional TDM & 49 & 11.5 \\
\hline P3.4 Antibiotics De-escalation & 7 & 1.6 \\
\hline Total & 427 & 100. \\
\hline Cause & $\mathbf{N}$ & Prol \\
\hline C1 Drug selection & 198 & 41.3 \\
\hline C1.1 Inappropriate drug according to guidelines/formulary & 45 & 9.4 \\
\hline C1.2 Inappropriate drug (within guidelines but otherwise contraindicated) & 42 & 8.8 \\
\hline C1.3 No indication for drug & 25 & 5.2 \\
\hline C1.4 Inappropriate combination of drugs, or drugs and herbal medications, or drugs and dietary supplements & 13 & 2.7 \\
\hline C1.5 Inappropriate duplication of therapeutic group or active ingredient & 12 & 2.5 \\
\hline C1.6 No or incomplete drug treatment in spite of existing indication & 42 & 8.8 \\
\hline C1.7 Too many drugs prescribed for indication & 5 & 1.0 \\
\hline C1.8 Necessary genetic testing before drug initiation & 14 & 2.9 \\
\hline C2 Drug form & 7 & 1.5 \\
\hline C2.1 Inappropriate drug form (for this patient) & 7 & 1.5 \\
\hline C3 Dose selection & 139 & 29.0 \\
\hline C3.1 Drug dose too low & 40 & 8.4 \\
\hline C3.2 Drug dose too high & 64 & 13.4 \\
\hline C3.3 Dosage regimen not frequent enough & 33 & 6.9 \\
\hline C3.4 Dosage regimen too frequent & 2 & 0.4 \\
\hline C4 Treatment duration & 65 & 13.6 \\
\hline C4.2 Duration of treatment too long & 65 & 13.6 \\
\hline C6 Drug use process & 4 & 0.8 \\
\hline C6.1 Inappropriate timing of administration or dosing intervals & 4 & 0.8 \\
\hline C8 Patient transfer related & 2 & 0.4 \\
\hline C8.3 Discharge/transfer information about medication incomplete or missing & 2 & 0.4 \\
\hline C9 Other & 64 & 13.4 \\
\hline C9.1 No or inappropriate outcome monitoring (incl. TDM) & 64 & 13.4 \\
\hline Total & 479 & 100. \\
\hline
\end{tabular}

Abbreviations: TDM, Therapeutic drug monitoring.

Table 2 Number of Drug Related Problems and Causes of All Medicines and Antibiotic Medicines of Pharmaceutical Consultation

\begin{tabular}{lllll}
\hline & All medicines & All medicines & Antibiotic & Antibioti \\
\hline Problem & N & Proportion, \% & N & Proporti
\end{tabular}




\begin{tabular}{lllll}
\hline & All medicines & All medicines & Antibiotic & Antibioti \\
\hline P1 Treatment effectiveness & 68 & 27.8 & 39 & 25.5 \\
P1.1 No effect of drug treatment & 4 & 1.6 & 3 & 2.0 \\
P1.2 Effect of drug treatment not optimal & 22 & 9.0 & 16 & 10.5 \\
P1.3 Untreated symptoms or indication & 40 & 16.3 & 19 & 12.4 \\
P1.4 Other & 2 & 0.8 & 1 & 0.7 \\
P2 Treatment safety & 143 & 58.4 & 99 & 64.7 \\
P2.1 Adverse drug event (possibly) occurring & 143 & 58.4 & 99 & 64.7 \\
P3 Treatment safety and cost-effectiveness & 15 & 6.1 & 12 & 7.8 \\
P4 Other & 19 & 7.8 & 3 & 2.0 \\
Total & $\mathbf{2 4 5}$ & $\mathbf{1 0 0 . 0}$ & $\mathbf{1 5 3}$ & $\mathbf{1 0 0 . 0}$ \\
Cause & $\mathbf{N}$ & $\mathbf{P r o p o r t i o n ,} \mathbf{\%}$ & $\mathbf{N}$ & $\mathbf{P r o p o r t i}$ \\
C1 Drug selection & 147 & 15.9 & 21 & 13.7 \\
C3 Dose selection & 3 & 59.8 & 114 & 74.5 \\
C4 Treatment duration & 13 & 1.2 & 2 & 1.3 \\
C6 Drug use process & 19 & 5.3 & 4 & 2.6 \\
C7 Drug-related side effects/Drug-induced diseases & 7.7 & 9 & 5.9 \\
C8 Other & 25 & 10.2 & 3 & 2.0 \\
Total & $\mathbf{2 4 6}$ & $\mathbf{1 0 0 . 0}$ & $\mathbf{1 5 3}$ & $\mathbf{1 0 0 . 0}$ \\
\hline
\end{tabular}

\section{FIGURE LEGENDS}

Fig 1 The flowchart for drug related problems classification during pharmaceutical intervention A flow chart was used to show the process of DRP classification using modified DRP classification system. Records removed $(n=11)$ are recommendations related to documentations or non-clinical issues.

Fig 2 Relationship between the drug related Problems and Causes identified during pharmaceutical intervention and consultation The size of the circle indicates how many times this DRPs or Cause was identified. An arrow pointing from P (Problems) to C (Causes) means the problem is caused by the corresponding cause and the number on the line indicates the frequency of this causal relationship.

a. During pharmaceutical intervention, the main Cause leading to adverse drug events (P2.1) was drug dose too high $(\mathrm{C} 3.2, n=58)$, contraindicated drug regimen $(\mathrm{C} 1.2, n=39)$, and no or inappropriate outcome monitoring (C9.1, $n=16)$. The main Causes of not optimal drug treatment (P1.2) was drug dose too low $(\mathrm{C} 3.1, n=37)$ and dosage regimen not frequent enough $(\mathrm{C} 3.3, n=32)$. Unnecessary drug treatment (P3.2) was mainly caused by drug duration too long $(\mathrm{C} 4.2, n=58)$.

b. During pharmaceutical consultation, the main Cause leading to P2.1 Adverse drug event was "C3 Dose selection" ( $n=94)$, followed by "C7 Drug related diseases" $(n=18)$ and "C1 Drug selection" ( $n=13)$, and the main Cause leading to "P1.3 Untreated symptoms or indications" was "C3 Dose selection" $(n=20)$ and "C1 Drug selection" $(n=16)$.

Fig 3 Comparison of drug related problems identified during pharmaceutical consultation and intervention A symmetrical bubble chart was used to describe the ratio of the proportion of DRPs identified during pharmaceutical consultation and intervention. The horizontal axis indicates the proportion of the corresponding Problem in all DRPs identified during pharmaceutical intervention. The vertical axis indicates the proportion of the corresponding Problem in all DRPs identified during pharmaceutical consultation. Four sub-category of DRPs were compared as they have the same definition in pharmaceutical intervention and consultation classification. DRPs not being compared mainly include P3.1 Problem with cost-effectiveness of the treatment and P3.2 Unnecessary drug-treatment. The size of the bubble is proportional to the ratio between $\mathrm{y}$ and $\mathrm{x}$ axis. The oblique line shown in the figure is a straight line with a slope of 1 . Bubbles above the line indicates that problem appears more frequently during medication consultation than pharmaceutical 
intervention. The order of ratio from high to low was 3.02 (P1.3 Untreated symptoms or indication; $16.3 \%$ / 5.4\%), 1.88 (P2.1 Adverse drug event (possibly) occurring; 58.4\%/ 31.1\%), 0.48 (P1.1 No effect of drug treatment; $1.6 \% / 3.3 \%$ ), and 0.35 (P1.2 Effect of drug treatment not optimal; 9\%/ 25.5\%).

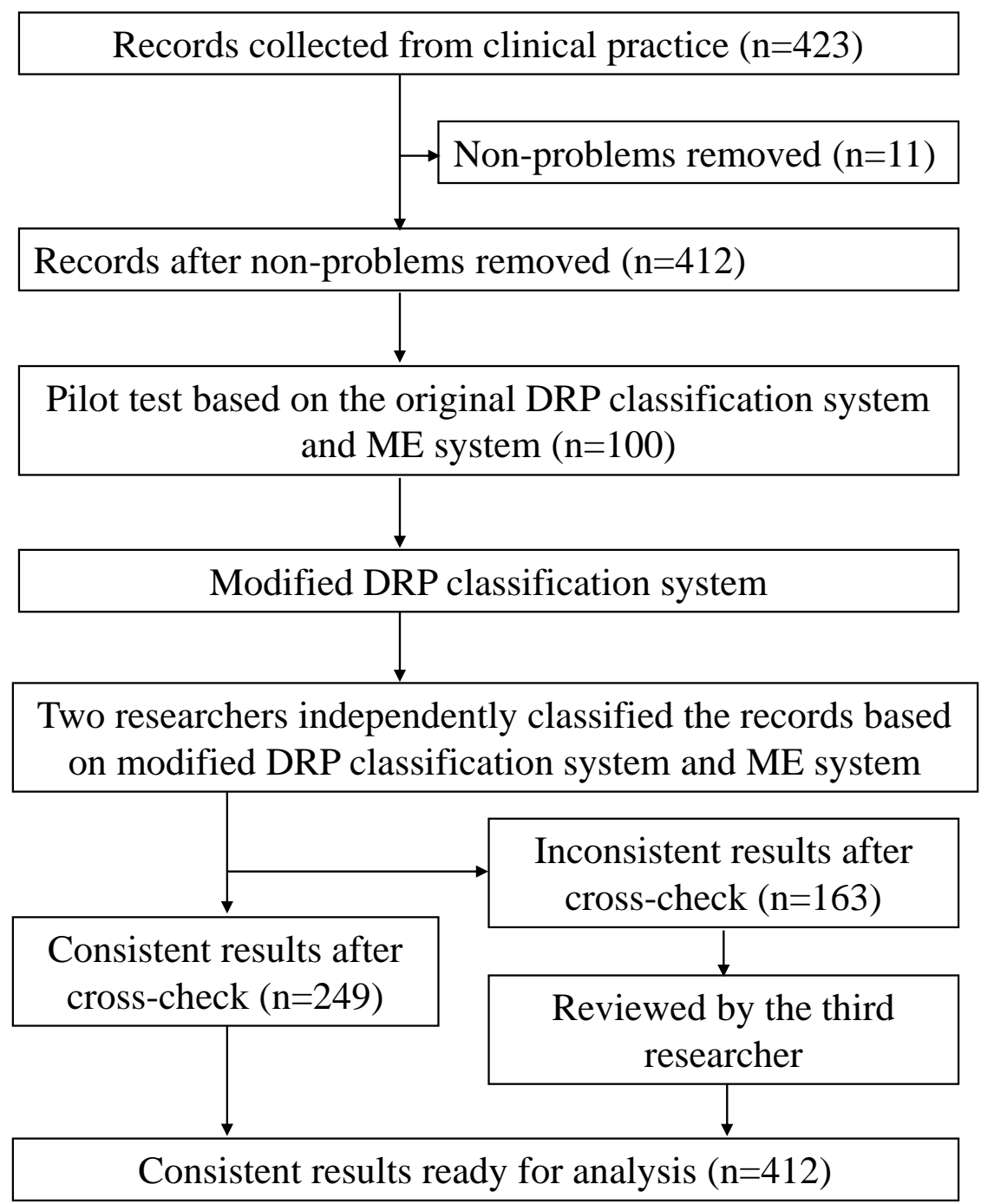




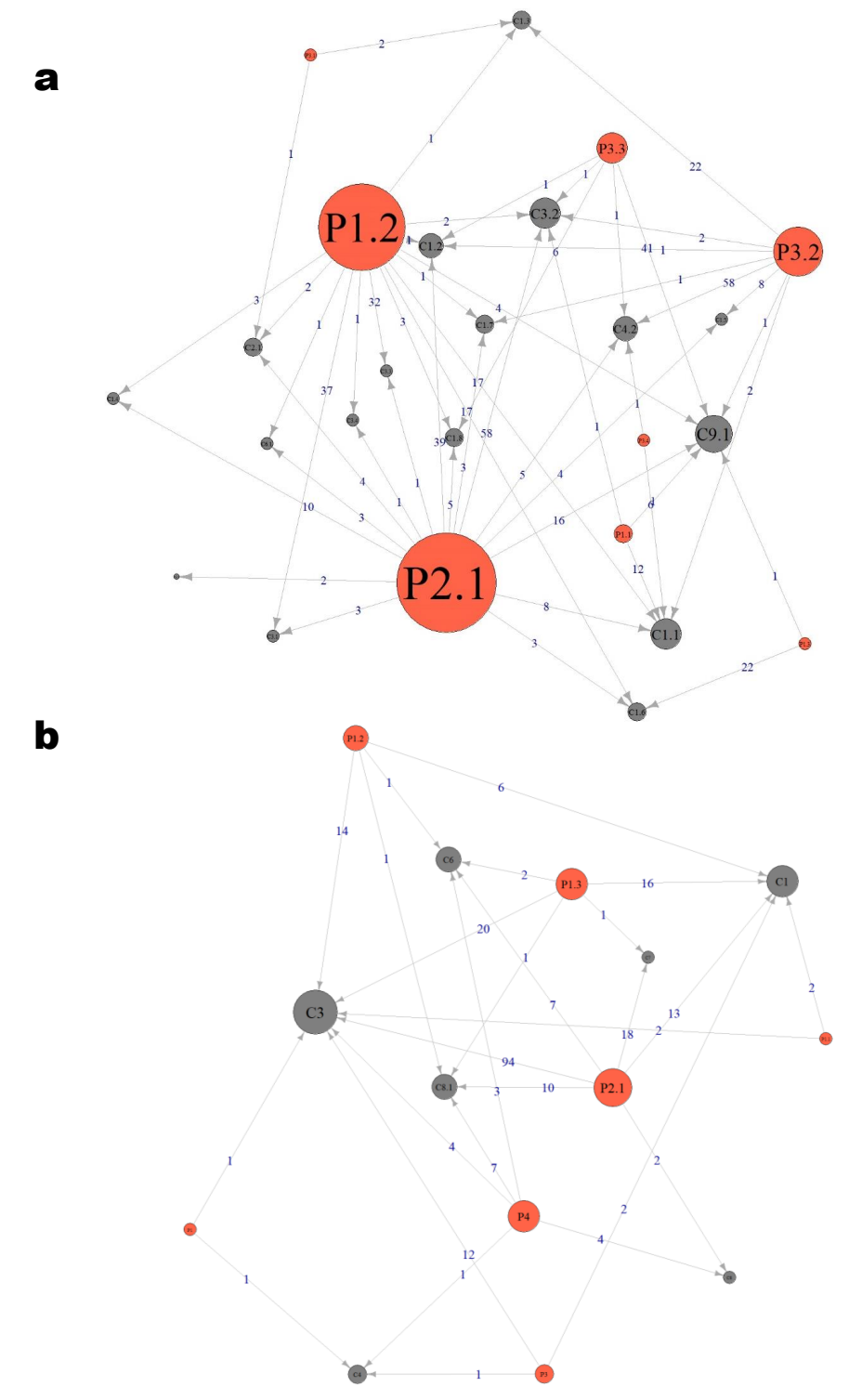




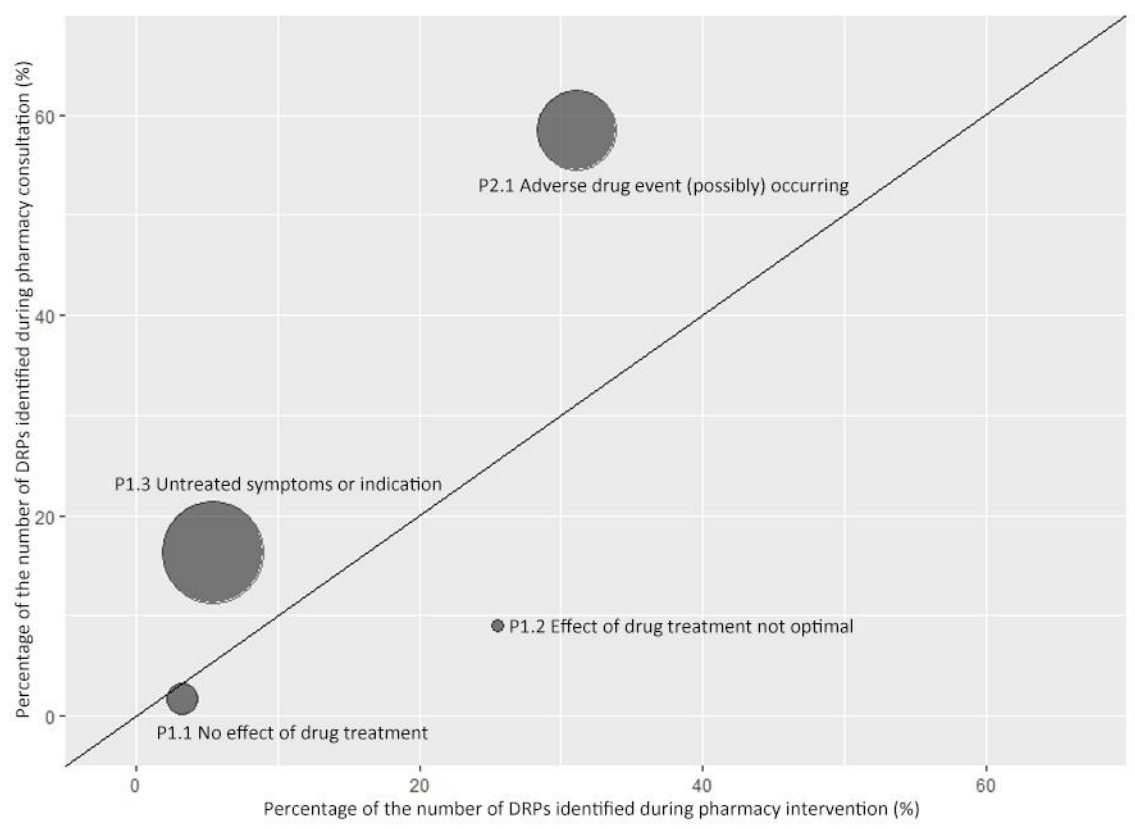

